\title{
Aanco Análise espacial da tuberculose em menores de 15 anos de idade e risco socioeconômico: um estudo ecológico na Paraíba, 2007-2016* \\ doi: 10.1590/\$1679-49742021000300006
}

\author{
Spatial analysis of tuberculosis in children under 15 years of age and socioeconomic risk: an \\ ecological study in Paraíba, Brazil, 2007-2016
}

\section{Análisis espacial de la tuberculosis en menores de 15 años y riesgo socioeconómico: un estudio ecológico en Paraíba, Brasil, 2007-2016}

\author{
Micheline da Silveira Mendes' 1 - orcid.org/0000-0001-7542-0702 \\ André Luiz Sá de Oliveira ${ }^{2}$ - (1) orcid.org/0000-0002-2483-550X \\ Lílian Maria Lapa Montenegro Pimentel ${ }^{3}$ - 0 orcid.org/0000-0002-4728-2995 \\ Tânia Maria Ribeiro Monteiro de Figueiredo ${ }^{4}$ - Đ orcid.org/0000-0001-6197-2936 \\ Haiana Charifker Schindler ${ }^{3}$ - 10 orcid.org/0000-0003-3863-4845 \\ 'Fundação Instituto Oswaldo Cruz, Departamento de Saúde Coletiva, Recife, PE, Brasil \\ ${ }^{2}$ Fundação Instituto Oswaldo Cruz, Núcleo de Estatística e Geoprocessamento, Recife, PE, Brasil \\ ${ }^{3}$ Fundação Instituto Oswaldo Cruz, Departamento de Imunologia, Recife, PE, Brasil \\ ${ }^{4}$ Universidade Estadual da Paraíba, Departamento de Enfermagem, Campina Grande, PB, Brasil
}

\section{Resumo}

Objetivo: Analisar a distribuição espacial da tuberculose em indivíduos menores de 15 anos de idade e fatores socioeconômicos na Paraíba, Brasil, 2007-2016. Métodos: Estudo ecológico, com dados do Sistema de Informação de Agravos de Notificação, sendo o município a unidade de análise. Realizou-se distribuição espacial da incidência, aplicou-se o método bayesiano empírico local e a estatística de Moran. Dados socioeconômicos foram cruzados, para identificação das áreas de prosperidade social. Resultados: Foram notificados 426 casos, com incidência média de 4,5/100 mil habitantes. 0 índice de Moran foi de 0,59 (p-valor=0,010). 0 Moran Map revelou concentração de casos em menores de 15 anos em 38 municípios com alta prioridade da atenção, em conglomerados nos padrões alto-alto e baixo-baixo, nas regiões leste e noroeste do estado, coincidindo com áreas de baixa prosperidade social. Conclusão: Houve clusters com maior transmissão da tuberculose, apontando áreas prioritárias para abordagem da tuberculose.

Palavras-chave: Estudos Ecológicos; Sistema de Informação em Saúde; Doenças Negligenciadas.

\footnotetext{
*Artigo derivado da dissertação de mestrado acadêmico intitulada 'Tuberculose em menores de 15 anos no estado da Paraíba: uma análise espaço-temporal no período de 2007 a 2016', apresentada por Micheline da Silveira Mendes junto ao Programa de Pós-Graduação em Saúde Pública do Curso de Mestrado Acadêmico em Saúde Pública/Instituto Aggeu Magalhães/Fundação Instituto Oswaldo Cruz, em 2019.
}

Endereço para correspondência:

Micheline da Silveira Mendes - Rua Enf. Ana Maria B. de Almeida, n 1217, Jd. Cidade Universitária, João Pessoa, PB, Brasil. CEP: 58052-270

E-mail:micheline_mendes@hotmail.com 


\section{Introdução}

A tuberculose é uma doença infectocontagiosa, causada pelo Mycobacterium tuberculosis. Ela acomete principalmente os pulmões (forma pulmonar), embora também possa atingir outros órgãos (forma extrapulmonar), e, apesar de tão antiga, conhecida, e curável, figura entre as doenças infecciosas que mais matam no mundo: em 2018, foram notificados 7 milhões de novos casos; e as notificações em menores de 15 anos de idade cresceram de menos de $400 \mathrm{mil}$, em 2015, para 523 mil em 2019., ${ }^{1,2}$

\section{Conhecer o panorama clínico da tuberculose na população mais jovem, assim como os padrões de distribuição no território e sua relação com fatores sociais, pode produzir informações úteis aos processos decisórios.}

A exemplo de outras doenças de importância epidemiológica, a tuberculose tem aspectos sociodemográficos associados a sua ocorrência e distribuição geográfica, pelo que se recomenda o desenvolvimento de estudos que visem conhecer mais profundamente esses fatores, para 0 apoio à tomada de decisões em políticas públicas de intervenção adequadas e oportunas. ${ }^{3}$

A infecção pelo $M$. tuberculosis está diretamente relacionada às condições de vida da população, sendo a pobreza um fator determinante para 0 consequente adoecimento ou agravamento de seus sintomas. A ocorrência da doença está associada a indicadores socioeconômicos, como condições de moradia, aglomerados urbanos, renda per capita, desemprego, educação, idade, acesso a serviços de saúde, alimentação e condições sanitárias, além da presença de comorbidades, especialmente quando estas levam a imunossupressão do indivíduo. ${ }^{4,5}$

A Organização Mundial da Saúde (OMS) reconhece a tuberculose como uma emergência de Saúde Pública e, nas últimas décadas, tem estimulado os países a inseri-la em políticas internacionais de saúde específicas para a doença, e, entre as diretrizes de gestão nacionais, incluir seu manejo quando afeta crianças e adolescentes. Para tanto, em 2006, a OMS publicou o Guidance for National Tuberculosis Programmes on the Management of Childhood Tuberculosis in Children, primeiro guia a abordar a tuberculose na infância, em todo o mundo.-8
A tuberculose em crianças ocorre, majoritariamente, nos países endêmicos para a doença, sendo pouco conhecida do ponto de vista epidemiológico, quando comparada às avaliações realizadas na população adulta. Contudo, o monitoramento da ocorrência da tuberculose na infância representa um indicador valioso de sua transmissão, especialmente em países de alta incidência. A identificação e exame dos contatos das crianças e adolescentes infectados constitui uma estratégia efetiva na identificação do caso-fonte. ${ }^{7}$

Conhecer o panorama clínico da tuberculose na população mais jovem, assim como os padrões de distribuição no território e sua relação com fatores sociais, demográficos e epidemiológicos, pode produzir informações úteis aos processos decisórios na construção e implementação de políticas públicas voltadas ao agravo na infância. ${ }^{9,10}$

A utilização de estatísticas espaciais em Saúde Pública e as relações com fatores socioambientais permite compreender as dimensões envolvidas nos estudos de nível ecológico e de nível individual da infecção, mediante o emprego de ferramentas de análise apropriadas, além de apoiar a vigilância do risco individual e a previsão de risco coletivo, de modo a entender - e controlar - processos endêmicos. ${ }^{9,10}$

Este artigo teve como objetivo analisar a distribuição espacial da tuberculose em indivíduos menores de 15 anos de idade e fatores socioeconômicos associados, no estado da Paraíba, Brasil, no perídio de 2007 a 2016.

\section{Métodos}

Estudo ecológico, com análise espacial dos casos novos de tuberculose em menores de 15 anos de idade notificados no período de 2007 a 2016.

0 estudo foi realizado na Paraíba, litoral oriental da região Nordeste do Brasil. 0 estado dispõe de área territorial de $56.468,435 \mathrm{~km}^{2}$, divididos em 223 municípios. Em 2010, a Paraíba contava com 3.766.528 habitantes, dos quais $27,5 \%$ eram menores de 15 anos de idade, apresentava índice de desenvolvimento humano municipal (IDHM) de médio desenvolvimento, índice de vulnerabilidade social (IVS) como de baixa vulnerabilidade e uma alta prosperidade social, com base no Censo Demográfico de 2010. ${ }^{11-14}$

Foram incluídos no estudo todos os casos notificados no Sistema de Informação de Agravos de Notificação (Sinan), com idade menor de 15 anos no momento do diagnóstico, de ambos os sexos e residentes do estado 
no período analisado; foram excluídos os casos com município de residência ignorado, e modo de saída por mudança de diagnóstico.

As variáveis do estudo foram obtidas das fichas de notificação compulsória, na base de dados do Núcleo de Doenças Endêmicas, da Secretaria de Estado da Saúde da Paraíba, coletadas em abril de 2018, após a limpeza de duplicidades e uma vez garantido anonimato dos indivíduos notificados (sem seu nome ou da mãe). Os dados populacionais foram obtidos em websites de domínio público, do Instituto Brasileiro de Geografia e Estatística (IBGE) e do Instituto de Pesquisa Econômica Aplicada, igualmente com base em dados do Censo 2010. ${ }^{13,14}$

As variáveis utilizadas para seleção dos casos foram: idade (em anos completos); ano (data de diagnóstico do caso); município de residência (no momento do diagnóstico); e população residente menor de 15 anos de idade (habitantes nessa faixa etária, por município). Para identificação da situação socioeconômica dos municípios, foram consideradas o IDHM e o IVS, utilizando-se os parâmetros de classificação do Programa das Nações Unidas para o Desenvolvimento (PNUD), em que o IDHM é classificado da seguinte maneira: muito baixo desenvolvimento $(0,000$ a 0,499$)$; baixo desenvolvimento $(0,500$ a 0,599$)$; médio desenvolvimento $(0,600 \mathrm{a} 0,699)$; alto desenvolvimento $(0,700 \mathrm{a}$ $0,799)$; e muito alto desenvolvimento ( $0,800 \mathrm{a} 1,000)$. 0 IVS classifica a vulnerabilidade da seguinte forma: muito baixa $(0,000$ a 0,200$)$; baixa $(0,201$ a 0,300$)$; média $(0,301$ a 0,400$)$; alta $(0,401$ a 0,500$)$; e muito alta $(0,500$ a 1,000). A sobreposição da faixa do IDHM à faixa do IVS permitiu identificar a prosperidade social dos municípios (Figura 1), utilizando-se, novamente, dados do Censo 2010 e os parâmetros do PNUD, segundo modelo proposto por Ferreira e Pinto. ${ }^{15}$

Foi calculado o coeficiente de incidência de casos novos na população de zero a 14 anos de idade (por 100 mil hab.), para a identificação de áreas de transmissão ativa da doença, e de concentração de casos (clusters). 0 cálculo médio de incidência para o período foi feito a partir da soma dos casos novos, dividida pelos 10 anos do estudo; o quociente dessa operação, por sua vez, foi dividido pela população de 0-14 anos de idade residente nos municípios (dados do Censo 2010), em seguida multiplicado por 100 mil. Logo, foi possível visualizar a distribuição espacial desse indicador no estado.

0 cálculo do indicador foi realizado com base na média do período total (2007 a 2016), visando corrigir flutuações aleatórias e proporcionar melhor estabilidade, principalmente em municípios com populações muito pequenas - por exemplo, com menos de 3 mil hab. Também foi calculado o indicador suavizado pelo método bayesiano empírico local, que utiliza informações das áreas vizinhas integrantes da mesma região, para estimar o risco da área. Os mapas temáticos dos coeficientes brutos e suavizados foram categorizados pelo método de quartis. ${ }^{16}$

Além da análise descritiva, utilizou-se: o Box Map (para visualizar a dependência espacial) e o índice de Moran I sobre o indicador suavizado, para avaliar a presença de dependência espacial global e áreas de significância estatística, e garantir a correção de valores extremos e de áreas silenciosas da doença. Dessa forma, foi possível medir a correlação de uma variável com ela mesma, no espaço, sendo seus valores estimados dentro de um intervalo de variação de $-1 \mathrm{a}+1$ : valores positivos e negativos desse intervalo indicam autocorrelação positiva e autocorrelação negativa, respectivamente; e valores próximos de zero, aleatoriedade espacial. ${ }^{17,18}$

A existência de correlação local foi avaliada pelo índice de Moran local (Local Index of Spacial Association), produzindo-se um valor específico para cada município, o que permitiu a comparação entre municípios vizinhos e a visualização de agrupamentos dos municípios com valores similares, e, por conseguinte, a identificação de padrões espaciais. A interpretação dos quadrantes, gerados a partir dessa técnica, demonstrou as seguintes correlações:

a) municípios com alta proporção do indicador, cercados por outros municípios também com altas proporções (alto-alto);

b) municípios com baixa proporção, cercados por municípios com baixa proporção do mesmo indicador (baixo-baixo), sugerindo pontos de associação espacial positiva; $\mathrm{e}$

c) municípios com alta proporção, cercados por municípios com baixa proporção desse indicador (alto-baixo), e municípios com baixa proporção, cercados de municípios com alta proporção do mesmo indicador (baixo-alto), sugerindo pontos de associação espacial negativa, representativos de áreas de transição.

Moran Maps foram usados para a representação espacial do diagrama de espelhamento de Moran, tendo a análise considerado o nível de significância menor que 5\%. Áreas de alto risco para transmissão ativa 
da doença e incidência de casos foram consideradas pela presença de municípios com elevados valores e vizinhos com a mesma característica.

A base georreferenciada utilizada, referente ao estado da Paraíba, obtida na página on-line do IBGE, tem formato vetorial, extensão shapefiles (.shp), sistema de projeção de coordenadas geográficas (lat,long) e Datum SIRGAS 2000. Para o processamento, análise, apresentação dos dados cartográficos, cálculo do indicador de autocorrelação espacial e produção dos mapas temáticos, foram utilizados os softwares de código aberto QGIS 2.14.8-Essen e TerraView versão 5.4.0.

0 projeto da pesquisa foi aprovado pelo Comitê de Ética em Pesquisa do Instituto Aggeu Magalhães/ Fundação Instituto Oswaldo Cruz em Pernambuco: Parecer $\mathrm{n}^{0}$ 2.655.809, emitido em 15 de maio de 2018.

\section{Resultados}

No período de 2007 até 2016 , foram diagnosticados e notificados, no estado da Paraíba, 426 casos novos de tuberculose em indivíduos menores de 15 anos de idade.

Os dados sobre o IDHM dos 223 municípios do estado foram distribuídos espacialmente. Demonstrouse que $152(68,2 \%)$ municípios foram de baixo desenvolvimento (Figura 2A). Na distribuição do IVS, observou-se a existência de 160 (71,7\%) municípios com vulnerabilidade alta ou muito alta, especialmente na zona leste do estado (Figura 2B). A prosperidade social foi considerada muito baixa em 135 (60,5\%) municípios paraibanos (Figura 2C).

$\mathrm{Na}$ faixa etária observada, a taxa média de incidência foi de 4,5/100 mil hab., com variação de 3,4 a 5,5/100 mil hab., considerando-se o conjunto do estado. No plano dos municípios, 91 (40,8\%) tiveram pelo menos um caso de tuberculose em menores de 15 anos, no período analisado, com incidência variável de 0,9 a 23,9/100 mil hab. (Figura 3A). 0 mapa suavizado, entretanto, revelou uma incidência esperada, entre 0,19 e 11,03/100 mil hab. As maiores incidências concentraram-se em municípios da zona leste do estado (Figura 3B).

A observação do Box Map identificou 86 municípios prioritários para monitoramento da ocorrência de casos de tuberculose (Figura 4A). Após a análise estatística, 0 índice de Moran global dos coeficientes suavizados, no período total, foi de 0,594 (p-valor=0,010). 0 Moran Map revelou concentração de casos em menores de 15 anos em 38 municípios com alta prioridade da atenção, em aglomerados de setores caracterizados por padrões alto-alto e baixo-baixo, identificados na zona leste e noroeste do estado respectivamente (Figura 4B).

\section{Discussão}

Entre os municípios da Paraíba, as áreas com maiores taxas de incidência de tuberculose na infância coincidiram com cenários de desigualdade social, apresentando IDHM avaliado baixo e IVS alto ou muito alto. Nenhum município foi avaliado com IDHM muito baixo ou muito alto, ou com IVS muito baixo.

Ao se analisarem os municípios com maior incidência média de tuberculose entre pessoas de idade inferior a 15 anos, no período selecionado para 0 estudo, observou-se elevada vulnerabilidade social, IDHM entre baixo e médio e prosperidade social baixa ou muito baixa, na maioria desses municípios. Esses dados diferem dos encontrados em estudos que analisaram o conjunto dos municípios do Brasil, nos anos 2000 e 2010, quando os resultados foram melhores na prosperidade social do país. Tal diferença justifica-se pela heterogeneidade da dimensão territorial brasileira, significativa entre suas grandes regiões. Por exemplo, a região Sul apresentou $84 \%$ de seus municípios nas duas faixas de mais alta prosperidade social, enquanto, na região Nordeste, apenas $3 \%$ se encontravam nesses estratos. ${ }^{15,19}$

Voltando ao presente estudo, no estado da Paraíba, a distribuição dos casos novos de tuberculose em menores de 15 anos de idade apontou uma heterogeneidade espacial, cuja análise identificou municípios sob elevado risco de transmissão ativa da doença. Observaram-se, ademais, aglomerados de municípios merecedores de atenção prioritária das intervenções. Entretanto, ao se utilizarem os métodos bayesiano e o índice de Moran local, verificou-se um número ainda maior de municípios com necessidade de intervenções em tuberculose focadas nos mais jovens.

Observou-se, também, a proximidade entre municípios com baixas taxas de incidência e outros com taxas elevadas, que não se apresentavam em aglomerados, ou ainda, municípios com notificações rodeados de outros sem nenhuma ocorrência da doença. Municípios com incidência bruta com valores iguais a zero podem indicar subnotificação de casos. Uma situação semelhante foi encontrada no 


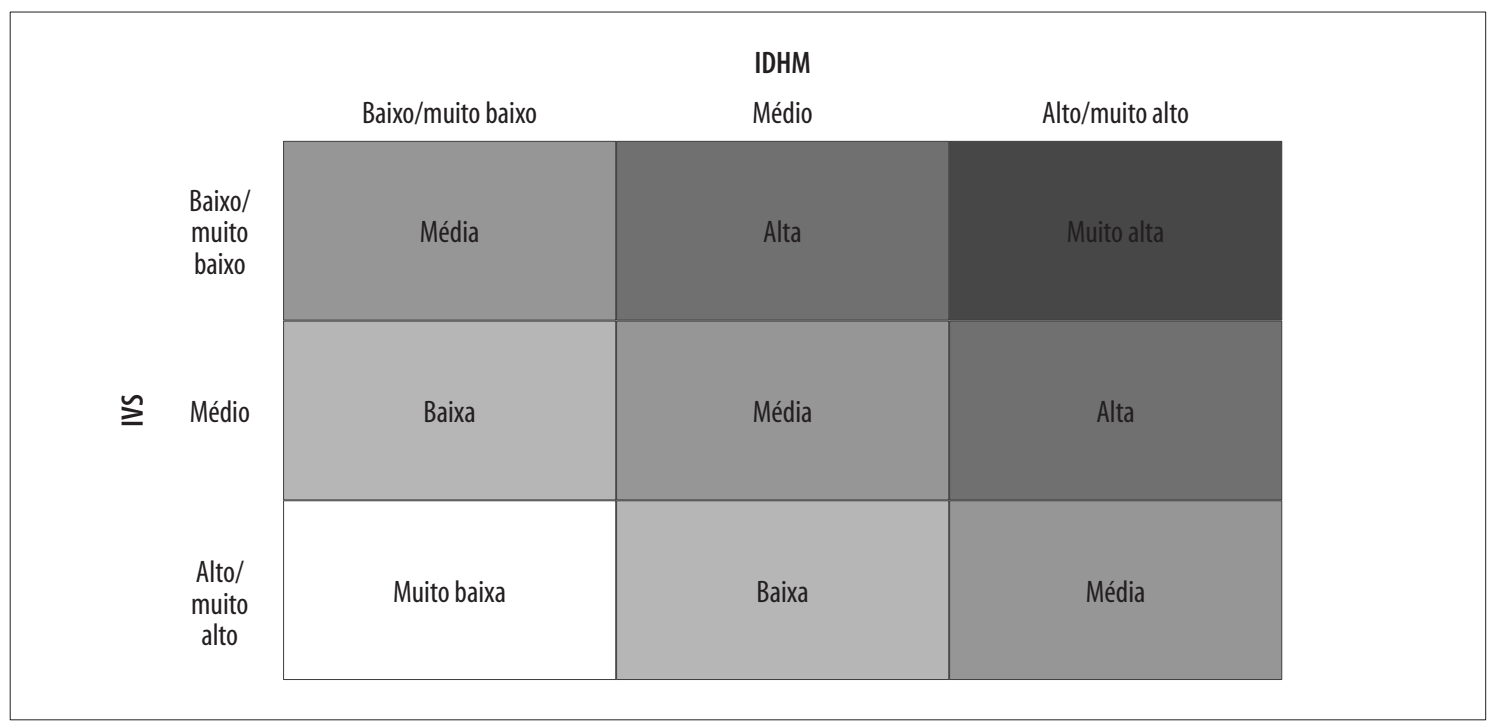

Fonte: Adaptado de Ferreira e Pinto. ${ }^{15}$

Figura 1 - Faixas de prosperidade social, a partir da relação entre as faixas do índice de desenvolvimento humano municipal (IDHM) e do índice de vulnerabilidade social (IVS)

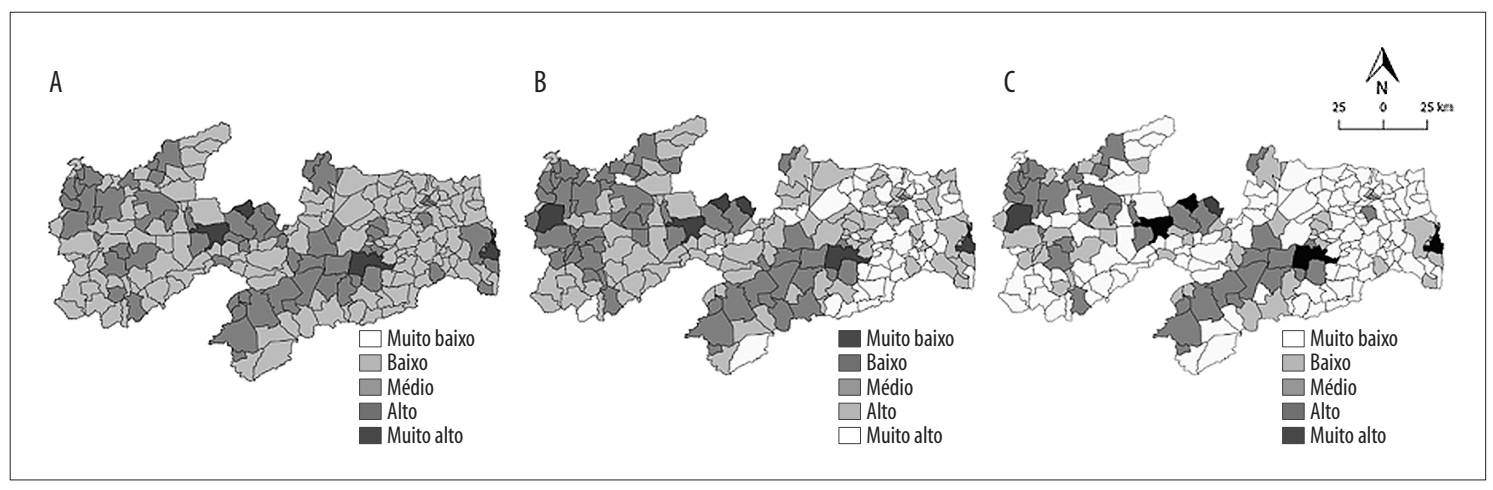

Figura 2 - Distribuição espacial do índice de desenvolvimento humano municipal (IDHM) (A), índice de vulnerabilidade social (IVS) (B) e prosperidade social (C) dos municípios da Paraíba

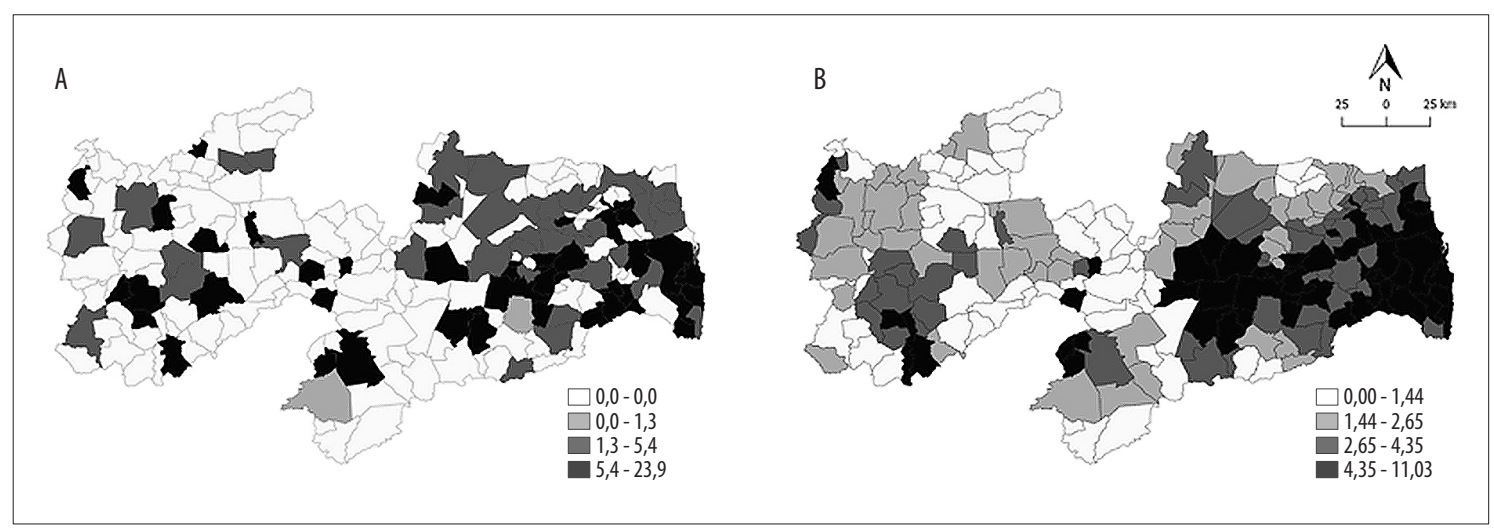

Figura 3 - Análise espacial do coeficiente de incidência de casos novos de tuberculose em menores de 15 anos de idade (por 100 mil hab.), coeficiente bruto (A) e coeficiente suavizado pelo método bayesiano empírico local (B), Paraíba, 2006-2017 

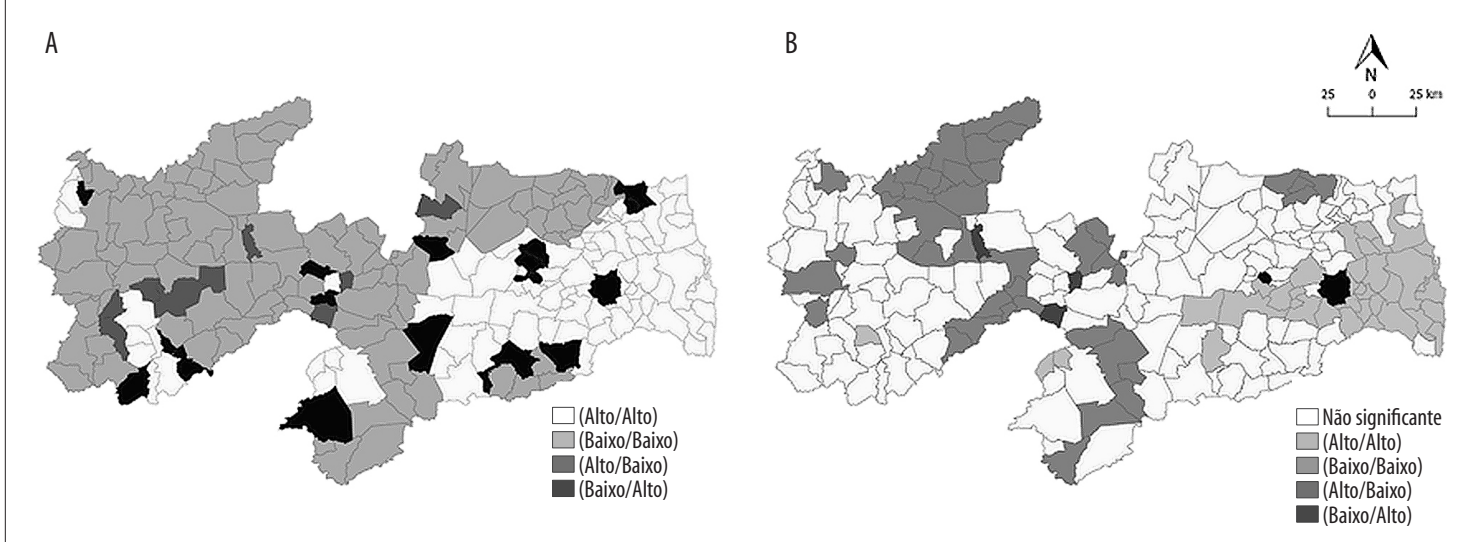

\section{Figura 4 - Análise espacial do coeficiente de incidência de casos novos de tuberculose em menores de 15 anos de idade (por 100 mil hab.), Box Map (A) e Moran Map (B), Paraíba, 2006-2017}

Espírito Santo, entre 2000 e 2007: municípios com zero de incidência de tuberculose infantil, limítrofes de municípios com incidência de 85 casos por 100 mil hab. na incidência bruta, sugerindo centralização do diagnóstico em algumas cidades e subnotificação nas cidades de origem. ${ }^{20}$

Vale ressaltar que a distribuição da incidência bruta revela um conjunto de municípios com altas taxas de incidência de tuberculose infantil em torno às regiões metropolitanas de João Pessoa e Campina Grande, maiores centros urbanos do estado. Esses achados fazem eco aos de Fortaleza, no período de 2000 a 2011, e de São Paulo, entre 2001 e 2010, quanto à distribuição espacial da doença. ${ }^{10,21}$

Apesar de as taxas brutas demonstrarem a distribuição espacial da doença, sua instabilidade e presença em locais com pequenas populações pode induzir a conclusões equivocadas. Para obtenção de resultados mais fidedignos, estudos têm-se utilizado de análise espacial a partir de taxas suavizadas. É o caso de pesquisas sobre a hanseníase que, ao se utilizarem do método bayesiano empírico local, alcançaram melhor compreensão das implicações espaciais devidas aos casos de municípios vizinhos, visualização do padrão espacial da doença, dos locais de risco e da influência de pequenas populações. ${ }^{17,20-23}$

Neste estudo, a análise da autocorrelação dos coeficientes suavizados possibilitou indicadores de incidência mais estáveis e a identificação de aglomerados de áreas mais críticas, abrangendo mais da metade dos municípios do estado, com clusters mais definidos na Zona da Mata e no Agreste paraibano. Este cenário reflete o quão grave se apresenta a tuberculose no estado, considerando-se que a área de casos de tuberculose na infância denota a transmissão recente da doença. ${ }^{10}$

Neste estudo, foi possível identificar áreas com significância estatística de risco da doença, contribuindo para o delineamento de áreas prioritárias para intervenção em saúde. Foram encontrados quatro clusters, o maior formado por 22 municípios, concentrados na zona leste do estado. Este achado demonstrou a associação espacial nessas áreas, e corroborou achados de outras investigações que utilizaram o índice de Moran I e encontraram áreas de autocorrelação espacial e clusters de transmissão ativa, evidenciados pelo Moran Map, identificando locais prioritários para o controle da doença. ${ }^{17,20,21}$

As possíveis limitações da pesquisa podem estar relacionadas ao uso de dados secundários do Sinan. Muito embora sejam dados oficiais, amplamente utilizados em trabalhos técnicos e científicos, estão sujeitos a inconsistências na quantidade, qualidade e processamento das informações. Diante disso, optouse por utilizar, como unidade de análise, o município, para evitar perdas de qualidade, posto que esse campo é de preenchimento obrigatório no Sinan. Não há como quantificar subnotificações, pelo que se espera serem reduzidas, uma vez que, no estado, condiciona-se o envio da medicação à notificação do caso. No que concerne 
às duplicidades, tampouco é possível quantificá-las, sequer conferir sua existência no banco de dados, embora a Secretaria de Estado da Saúde já houvesse disponibilizado os dados após eliminar duplicidades e saídas por erro diagnóstico. Outra possível limitação do estudo residiria no fato de a situação epidemiológica da tuberculose nessa faixa etária ser bastante reduzida, proporcionalmente, frente às demais idades.

Foi realizada a distribuição espacial da tuberculose em indivíduos menores de 15 anos de idade na Paraíba, identificando-se clusters de incidência no estado, e confirmou-se que a análise espacial pode ser um importante instrumento na identificação de locais de casos de tuberculose infantil. Nesse contexto, o estudo pode ser considerado pioneiro em analisar a ocorrência da tuberculose entre menores de 15 anos de idade e sua distribuição espacial em um estado do Nordeste brasileiro.

Os resultados obtidos indicam que mais pesquisas devam se realizar, inclusive em outros estados do Brasil, no sentido de contribuir com o monitoramento de áreas de transmissão ativa da doença e criar subsídios

\section{Referências}

1. Cano APG, Romaneli MTN, Pereira RM, Tresoldi AT. Tuberculose em Pacientes Pediátricos: Como tem sido feito o diagnóstico? Rev Paul Pediatr.2017; 35(2):16570. doi: https://doi.org/10.1590/1984-

0462/;2017;35;2;00004.

2. World Health Organization. Global tuberculosis report: 2020. Geneva: WHO; 2020.297 p.

3. Almeida MG, Barbosa DRM, Almeida DFS. Epidemiologia e distribuição espacial da tuberculose multirresistente (TBMR) no Brasil notificada através do SINAN, 2008-2012. Rev Epidemiol Control Infec 2013;3(4):117-22. doi: http://dx.doi.org/10.17058/ reci.v3i4.3564

4. Pereira AGL, Medronho RA, Escosteguy CC, Valencia LIO, Magalhães MAF. Distribuição espacial e contexto socioeconômico da tuberculose, Rio de Janeiro, Brasil. Rev. Saude Publica. 2015; 49(48). doi: https:// doi.org/10.1590/S0034-8910.2015049005470.

5. Santos MLSG, Vendramini SHF, Gazetta CE, Oliveira SAC, Villa TCS. Pobreza: caracterização socioeconômica da tuberculose. Rev Lat-Am Enfermagem. 2007; 15(n.spe):762-7. doi: https://doi. org/10.1590/\$0104-11692007000700008. para o Programa Nacional de Controle da Tuberculose e suas ações com foco na infância e adolescência. Futuros estudos também podem auxiliar o planejamento de atividades de base territorial local e o monitoramento técnico, político e operacional das ações de combate à doença e promoção da saúde, tendo a tuberculose na infância e adolescência como evento sentinela de transmissão recente da infecção.

\section{Contribuição dos autores}

Mendes MS e Schindler HC participaram da concepção e planejamento do estudo, análise e interpretação dos dados e redação final do manuscrito. Oliveira ALS participou do planejamento do estudo, análise e interpretação dos dados e revisão crítica do manuscrito. Pimentel LMLM e Figueiredo TMRM participaram da revisão do manuscrito. Todos os autores aprovaram a versão final do manuscrito e declaram-se responsáveis por todos os aspectos do trabalho, garantindo sua precisão e integralidade.
6. Duarte R, Silva DR, Rendon A, Alves TG, Rabahi MF, Centis R et al. Eliminação da tuberculose na América Latina: considerações. J Bras Pneumol. 2018;44(2):73-6. doi: https://doi. org/10.1590/s1806-37562017000000449.

7. World Health Organization. Guidance for national tuberculosis programmes on the management of tuberculosis in children. 2nd ed. Geneva, WHO;2014.146 p.

8. Sant'anna CC, Hijjar MA. Recente contribuição da Organização Mundial de Saúde para o controle da tuberculose na infância. Rev Saude Publica. 2007; 41(sppl.1):117-20. doi: https://doi.org/10.1590/ S0034-89102007000800016.

9. Silva LB Análise Espacial dos casos de tuberculose na Rocinha, no período de 2007 a 2013, Rio de Janeiro. [dissertação]. [Rio de Janeiro]: Universidade Federal do Estado do Rio de Janeiro.

10. Venâncio TS, Tuan TS, Nascimento LFC. Incidência de tuberculose em crianças no estado de São Paulo, Brasil, sob enfoque espacial. Cienc Saude Colet 2015; 20(5):1541-47. doi: http://dx.doi.org/10.1590/141381232015205.14672014 . 
11. Brandão ICA, Martiniano CS, Monteiro AI, Marcolino EC, Brasil SKD, Sampaio J. Análise da organização da rede de saúde da Paraíba a partir do modelo de regionalização. Rev Bras Cienc. Saude. 2012; 16(3):347-52.

12. Instituto Brasileiro de Geografia e Estatística. Coordenação de população e indicadores sociaisCOPIS: projeção da população. Brasília, DF: IBGE; [data desconhecida] [acesso 17 nov.2018]. Disponível em: https:/www.ibge.gov.br/ geociencias/ organizacao-do-territorio/malhas-territoriais.html.

13. Costa MA, Marguti BO, editores. . Atlas da vulnerabilidade social nos municípios brasileiros. Brasília, DF: Ipea: 2015 [acesso 17 nov. 2018]. Disponível em: https://www.ipea.gov.br/portal/index. php?option=com_content\&amp; view=article\&amp; $\mathrm{id}=26118$.

14. Instituto Brasileiro de Geografia e Estatística [homepage na internet]. Geociências, organização do território, malhas territoriais. Brasília, DF: IBGE; [data desconhecida] [acesso 17 nove. 2018]. Disponível em: http:www.ibge.gov.br

15. Ferreira GS, Pinto CVS. Desenvolvimento humano, vulnerabilidade e prosperidade social no Rio Grande do Sul: uma visão inter e intrarregional por meio dos Coredes. In: Marguti BO, Costa MA, Pinto CVS, organizadores. Brasília, DF: Ipea; 2017:297-332.

16. Assunção RM, Barreto S, Guerra HL, Sakurai E. Mapas de taxas epidemiológicas: uma abordagem Bayesiana. Cad Saude Publica. 1998; 14(4):713-23. doi: https:// doi.org/10.1590/S0102-311X1998000400013.
17. Barbosa CC. Padrões espaciais dos casos novos da hanseníase no estado de Pernambuco, Brasil, 2016 [dissertação]. [Recife]: Instituto Aggeu Magalhães, Fundação Oswaldo Cruz. 2017.

18. Santos SM, Souza WV. organizadores. Introdução à estatística espacial para a saúde pública.. Brasília, DF: MS, Fundação Oswaldo Cruz, 2007.

19. Costa MA, Marguti BO. Atlas da vulnerabilidade social nos municípios brasileiros. IPEA (Instituto de Pesquisa Econômica Aplicada). [internet] 2015.

20. Sales CMM, Figueiredo TAM, Zandonade E, Maciel ELN. Análise espacial da tuberculose infantil no estado do Espírito Santo, 2000 a 2007. Rev Soc Bras Med Trop. 2010;43(4):435-9. doi: http://dx.doi. org/10.1590/S0037-86822010000400020.

21. Lima MS. Padrões espaço-temporais da mortalidade relacionada à coinfecção tuberculose e HIV/AIDS no Brasil, 2000-2011 [dissertação]. [Fortaleza]: Faculdade de Medicina, Universidade Federal do Ceará. 2015. 116 f.

22. Lorena DM, Martins-Melo FR, Brito AL, Alencar CH, Heukelbach J. Spatial patterns of leprosy in a hyperendemic state in Northern Brazil, 2001-2012. Rev Saude Publica.2015; 49:84. doi: https://doi. org/10.1590/S0034-8910.2015049005866.

23. Magalhães MAFM, Medronho RA. Análise espacial da Tuberculose no Rio de Janeiro no período de 2005 a 2008 e fatores socioeconômicos associados utilizando microdado e modelos de regressão espaciais globais. Cienc Saude Colet, 2017;22(3):831-40. doi: https:// doi.org/10.1590/1413-81232017223.24132015. 


\section{Abstract}

Objective: To analyze spatial distribution of tuberculosis in individuals under 15 years old and socioeconomic factors in Paraíba, Brazil, 2007-2016. Methods: This was an ecological study based on data from the Notifiable Health Conditions Information System (SINAN), taking each municipality to be a unit of analysis. Spatial distribution of incidence was performed, the local empirical Bayesian method and Moran's I were applied. Socioeconomic data were crossed-checked to identify areas of social prosperity. Results: 426 cases were notified, with average incidence of 4.5/100,000 inhabitants. Moran's I was $0.59(p=0.010)$. The Moran Map revealed concentration of cases in children under 15 in 38 high priority municipalities, in clusters with high-high and low-low patterns, in the east and northwest of the state, coinciding with areas of low social prosperity. Conclusion: There were clusters with greater tuberculosis transmission, indicating priority areas for addressing tuberculosis.

Keywords: Ecological Studies; Health Information Systems; Neglected Diseases.

\section{Resumen}

Objetivo: Analizar la distribución espacial de la tuberculosis en menores de 15 años y los factores socioeconómicos en Paraíba, Brasil, 2007-2016. Métodos: Estudio ecológico, con datos de Sistema de Información de Agravamientos de Notificación, considerando el municipio como unidad de análisis. Se realizó la distribución espacial de la incidencia, se aplicó el método empírico local Bayesiano y la estadística de Moran. Se cruzaron datos socioeconómicos para identificar áreas de vulnerabilidad social. Resultados: Se notificaron 426 casos, con una incidencia promedio de 4,5/100 mil habitantes. El indice de Moran = 0,59 $(p=0,010)$. El Mapa de Moran reveló una concentración de casos en menores de 15 años en 38 municipios con alta prioridad de atención, en conglomerados en los patrones alto-alto y bajo-bajo, en las regiones este y noroeste del estado, coincidiendo con áreas de baja prosperidad social. Conclusión: Existían clusters con mayor transmisión de tuberculosis, lo que indica áreas prioritarias para abordar la tuberculosis.

Palabras clave: Estudios Ecológicos; Sistema de Información Sanitaria; Enfermedades Desatendidas.

Recebido em 06/12/2020

Aprovado em 05/02/2021

Editor associado: Bárbara Reis-Santos - — orcid.org/0000-0001-6952 0352 Editora científica:Taís Freire Galvão - @o orcid.org/0000-0003-2072-4834 Editora geral: Leila Posenato Garcia - @ orcid.org/0000-0003-1146-2641 